\title{
CONSIDERATIONS ON BEAM QUALITY CONTROL IN MIT X-RAY FEL
}

\author{
D. Wang, W. Graves, D.F. Wang, T. Zwart, Bates Laboratory, MIT, Middleton, MA 01949, \\ P. Emma, J. Wu, SLAC, Stanford, CA 94309, G. Huang, LBL, Berkeley, CA 94720
}

\section{Abstract}

The x-ray FEL at MIT[1] is one example of a design for a new generation linac-based light source. Such a new machine requires very high quality electron beams. Besides the usual requirements on beam parameters such as emittance, energy spread, peak current, there are new challenges emerging in the design studies, e.g., the arrival timing of electron beam must reach precision below tens of femtoseconds level to ensure the laser seed overlaps the desired sections of electron bunch in the multiple-stage HGHG process. In this paper we report the progress on design optimization towards high quality and low sensitivity beams.

\section{INTRODUCTIONS}

The proposed MIT x-ray laser incorporates design features which take advantage of many recent developments. It blends proven technologies into a powerful new instrument that combines the high power, coherence, and ultrashort timescale probe of a laser with the energy reach and spatial resolution of synchrotron $\mathrm{x}$ rays. It is a primary goal to integrate the instruments and experimental methods from the laser and synchrotron radiation communities at the earliest stages of design. Integrated high-harmonic generation laser technology will seed the electron beam and generate photon beams with high longitudinal coherence and pulse lengths significantly below 100 femtoseconds, perhaps below 1 femtosecond. The FEL itself will use the high gain harmonic generation (HGHG) method to produce multiple harmonics of the tunable input seed. The output radiation has the full longitudinal and transverse coherence and stability of the seed laser, providing substantial improvement over performance based solely on SASE. Table 1 summarizes the overall design parameters of MIT $\mathrm{X}$-ray FEL machine.

Table 1 Beam parameters of MIT X-ray FEL

\begin{tabular}{ll}
\hline \hline Final Beam energy & $4 \mathrm{GeV}$ \\
Bunch length & $100-1000 \mathrm{fs}$ \\
Normalized emit. & $0.5-2 \mathrm{~mm} . \mathrm{mrad}$ \\
Charge per bunch & $200-1000 \mathrm{pc}$ \\
Energy spread & $0.01 \%$ (sliced) \\
Peak current & $\sim 1-2 \mathrm{kA}$ \\
\hline \hline
\end{tabular}

The major components of the linac are the superconducting electron linac of length $\sim 300 \mathrm{~m}$, plus undulator tunnels and experimental halls. The production of $\mathrm{x}$-ray laser pulse begins with generation of the electron beam in the RF photoinjector. The photoelectrons are produced by a conventional laser striking the photocathode contained in a high field RF cavity, producing $200 \mathrm{pC}$ to $1 \mathrm{nC}$ pulses that are between 10 and 25 picoseconds long, depending upon operation modes. The superconducting linac will accelerate electron beam to up to $4 \mathrm{GeV}$ energy. At about $200 \mathrm{MeV}$ and $900 \mathrm{MeV}$, the beam enters the first and second magnetic chicane, which compress the pulse length to between 100 and 1000 femtoseconds and increase the bunch current from a few tens of amps to a few thousand amps.

\section{BEAM AND SEED LASER SYNCHRONIZATION REQUIREMENT}

In FEL production with laser seeds, the arrival time of electron beam must be very precise to overlap the seed. Plus, the pump lasers for many experiments also need to be synchronized with FEL pulses. So arrival times of those three sorts of very short pulses must be very precise,

- Electron bunch (different sections of same bunch may be used in different stages)

- Seed lasers for all stages

- Pump lasers at experiment stations.

The typical bunch length is between 100 and $1000 \mathrm{fs}$ in new FEL facilities. The requirement of arrival timing of beam is even tougher in multi-stage HGHG process in which a single electron bunch may be used section by section in different stages. Figure 2 shows the time structure of an electron bunch in a typical multi-stage HGHG process.

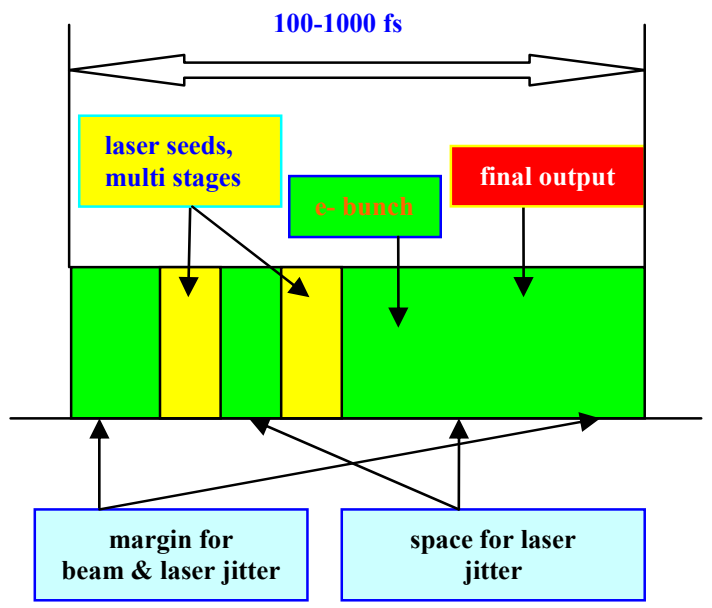

Figure 1: Arrival timing requirement of electron bunch and laser seed in the multi-stage HGHG process.

To ensure that the electron bunch and laser meet each other, the arrival times of both beams must be very precise, i.e., the arrival timing jitters are significantly shorter than the electron bunch length. When bunch length is between 100-200 femtoseconds, the arrival 
timing needs to be as precise as $\sim 20$ fs to efficiently utilize the full length of the electron bunch.

\section{DESIGN OPTIMIZATIONS INCLUDING JITTER REQUIREMENTS}

The unprecedented precision requirement for arrival time of electron bunches has become one of the most challenging issues in design studies of MIT $x$-ray FEL facility. For comparison two major ongoing x-ray FEL projects (LCLS and TESLA XFEL) [2] [3] have studied tolerance budgets for $<12 \%$ rms peak-current jitter and $0.1 \%$ rms final electron energy jitter. By applying these tolerance budgets the resulting arrival time jitters of beam are at about $100 \mathrm{fs}$ level. Our recent studies show that to limit arrival timing jitter to $20 \mathrm{fs}$ level the requirements for RF phase and amplitude control can be quite strict. Hence in our recent design studies the arrival jitter issue is treated as an integral part of the optimization.

The arrival time jitter of electron bunch can be caused by various errors in accelerators. The analytical estimates work mainly for simple cases, for example, for a single bunch compressor or linac section.

$$
\Delta t_{i}=\Delta t_{i-1}+\frac{1}{c}\left(\frac{\Delta E}{E}\right)_{i} R_{56 i}^{\prime}
$$

and

$$
\begin{gathered}
R_{56 i}^{\prime}=R_{56 i}+2 T_{566 i}\left(\frac{\Delta E}{E}\right)_{i} \\
\left(\frac{\Delta E}{E}\right)_{i}=\frac{E_{i-1}\left(\frac{\Delta E}{E}\right)_{i-1}-\frac{\Delta N}{N} \frac{\Delta E_{\text {loadi }}}{E_{i}}+}{\left(1-\frac{E_{i-1}}{E_{i}}\right)\left[\frac{\cos \left(\varphi_{i}+\Delta \varphi+2 \pi c \Delta t_{i-1} / \lambda_{i}\right.}{\cos \left(\varphi_{i}\right)}-1\right]} \\
\Delta E_{\text {lood }_{i}} \approx \frac{1}{2} E_{i} \Delta z_{i} k_{w_{i}}
\end{gathered}
$$

i denotes the current section of the linac, i-1 the previous section, $\Delta \mathrm{t}$ the timing error, $\Delta \mathrm{E} / \mathrm{E}$ the energy spread, $\Delta \mathrm{E}_{\text {load }}$ the wake loading, $\varphi$ the RF phase in linac, $\mathrm{N}$ the number of electrons.

In order to better understand the issue a fast optimization code [4] based on above simplified theoretical models is used. The sensitivities of arrival timing, as well as beam energy and peak current, to the major components in the linac are calculated in each iteration of the optimization. Figure 3 shows an example of the second chirp linac section. The initial laser pulse length on the cathode is about $25 \mathrm{ps}$. The bunch charge is $1 \mathrm{nC}$. Table 2 and 3 show two sets of machine parameters that both fulfill the general beam quality requirements shown in Table 1 but have different sensitivities to the errors.

The sensitivities of beam quality to different parameters are calculated. See Table 4 and 5. Besides sensitivities to the change in energy $(0.1 \%)$ and peak current $(12 \%)$ the arrival timing requirement $(20 \mathrm{fs})$ is also calculated and shown in the $5^{\text {th }}$ column. The monitored parameters include initial charge variation, RF phase jitters in gun, four major linac sections and the $3^{\text {rd }}$ harmonic cavity, Rf amplitude jitters in linac sections and the $3^{\text {rd }}$ harmonic cavity. It is clear that the arrival timing jitter requirement has become the dominant factor. On the other hand one can see that the sensitivity can be significantly improved by optimizing the linac parameters. For example the requirement of RF phase control level in section 2 (the most sensitive one) is relaxed by a factor of two in the latter case.

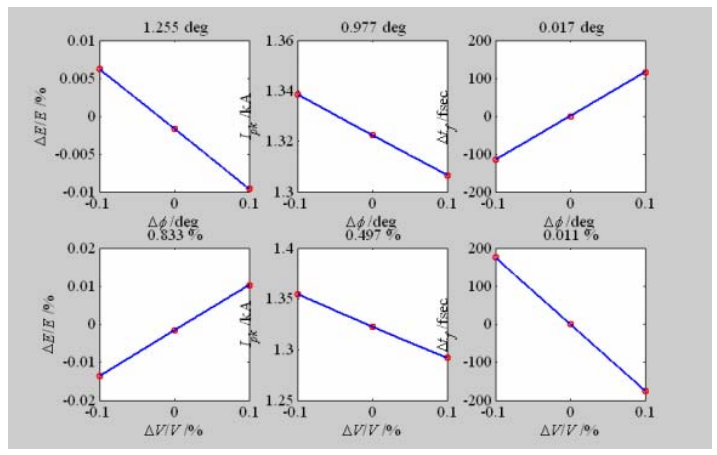

Figure 2: sensitivities of beam quality to jitters in each section of linac (shown here is $2^{\text {nd }}$ chirp linac section).

Tables 4 and 5: Comparison of sensitivities of beam quality to various jitters in different sections of the linac

\begin{tabular}{|l|c|c|c|c|}
\hline & & $\mathrm{dE} / \mathrm{E}$ & $\mathrm{I}$ peak & $\mathrm{dt} / \mathrm{t}$ \\
\hline Gun & phase & 17.7 & 1.2 & 1.2 \\
\hline & $\mathrm{dQ} / \mathrm{Q}$ & $107 \%$ & $19 \%$ & $3 \%$ \\
\hline Linac 1 & $\mathrm{phase}$ & 2.8 & 0.062 & 0.499 \\
\hline & $\mathrm{dV} / \mathrm{V}$ & $0.7 \%$ & $0.18 \%$ & $0.006 \%$ \\
\hline Linac 2 & phase & 0.66 & 0.034 & 0.006 \\
\hline & $\mathrm{dV} / \mathrm{V}$ & $0.55 \%$ & $0.37 \%$ & $0.005 \%$ \\
\hline 3rd har. & $\mathrm{phase}$ & 3.9 & 0.071 & 0.842 \\
\hline & $\mathrm{dV} / \mathrm{V}$ & $2.5 \%$ & $0.6 \%$ & $0.02 \%$ \\
\hline Linac 3 & $\mathrm{phase}$ & 1.1 & 1.4 & 0.02 \\
\hline & $\mathrm{dV} / \mathrm{V}$ & $0.47 \%$ & $1.5 \%$ & $0.009 \%$ \\
\hline Linac 4 & $\mathrm{phase}$ & 2.8 & - & - \\
\hline & $\mathrm{dV} / \mathrm{V}$ & $0.13 \%$ & - & - \\
\hline
\end{tabular}

\begin{tabular}{|l|c|c|c|c|}
\hline & & $\mathrm{dE} / \mathrm{E}$ & $\mathrm{I}$ peak & $\mathrm{dt} / \mathrm{t}$ \\
\hline Gun & phase & 12.6 & 1.3 & 1.9 \\
\hline & $\mathrm{dQ} / \mathrm{Q}$ & $161 \%$ & $32 \%$ & $3.5 \%$ \\
\hline Linac 1 & phase & 3.7 & 0.068 & 0.518 \\
\hline & $\mathrm{dV} / \mathrm{V}$ & $1.1 \%$ & $0.41 \%$ & $0.007 \%$ \\
\hline Linac 2 & phase & 1.67 & 0.046 & 0.012 \\
\hline & $\mathrm{dV} / \mathrm{V}$ & $0.79 \%$ & $1.46 \%$ & $0.005 \%$ \\
\hline 3rd har. & phase & 4.1 & 0.087 & 1.0 \\
\hline & $\mathrm{dV} / \mathrm{V}$ & $3.9 \%$ & $1.7 \%$ & $0.025 \%$ \\
\hline Linac 3 & phase & 1.5 & 1.7 & 0.045 \\
\hline & $\mathrm{dV} / \mathrm{V}$ & $0.54 \%$ & $3.0 \%$ & $0.016 \%$ \\
\hline Linac 4 & $\mathrm{phase}$ & 2.5 & - & - \\
\hline & $\mathrm{dV} / \mathrm{V}$ & $0.13 \%$ & - & - \\
\hline
\end{tabular}

\section{FEEDBACK IN LINAC SYSTEM}

To minimize variations of different beam parameters due to errors in RF phases, amplitudes, drive lasers timings, etc., feedback systems are designed for new generation FEL facilities like LCLS. The rms arrival timing can be controlled to $150 \mathrm{fs}$ level [7] which is 
already very good for a SASE machine. The in-depth analysis points out that drive laser timing jitter can not be 'compressed' like other errors while going through the linac system hence it mostly sets the limit of this kind of feedback[8]. In case of HGHG machine, in particular when fresh bunch scheme is applied, the arrival timing jitter must be better then a fraction of bunch length, i.e., a few tens of femto-seconds. Besides pushing RF and laser system technology to the new limits one needs to think other ways to solve the problem.

\section{ARRIVAL TIMING FEEDBACK AFTER LINAC}

A useful and practical feedback scheme is proposed here to achieve the precise control of arrival timing of beam. It is noticed that the path length of the relativistic electron beam corresponding to femto-second time period is quite small. For example, $10 \mathrm{fs}$ is equivalent to 3 micrometers. This fact implies two issues:

1) errors of orbital motions of beams need to be small

2) precise arrival timing can be controlled through tiny path length changes caused by transverse orbital motions

In fact one could utilize this feature to accurately adjust the arrival timing of the beam.

The feedback system is primarily considered to be independent from that of linac feedback system because the feedback systems in linac involve too many beam parameters. Relationships between beam emittance, energy spread, bunch length, arrival timing, etc. and RF phase, amplitude, offset(hence wakefields), etc., are so complicated that it is hard to control a single parameter while keeping others unchanged.

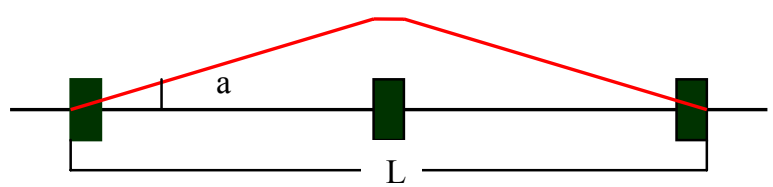

Figure 3: Timing adjustment through orbit bumps.

Another reason for an independent arrival timing feedback system is that each beam line may have specific needs to synchronize beam and seed laser or pump lasers.

For the simplest case of a closed orbit local bump, the path length difference due to a transverse kick with angular change of a is about $L a^{2}$ (when a is small and $\mathrm{L}$ is the length of bump). For a $10 \mathrm{~m}$ bump, $1 \mathrm{mrad}$ bending angle will roughly result in $30 \mathrm{fs}$ shift in arrival timing. The exact expression of path length change is also straight forward. If dipole field length is $0.2 \mathrm{~m}$, this corresponds $\sim 170$ gauss magnetic field for $1 \mathrm{GeV}$ electron beam. Such a bending scheme can be realized in many ways:

- ferrite magnets,

- corrector coils,

- $\quad$ strip lines,

- deflecting cavities, etc., depending upon the repetition rate (for example, $120 \mathrm{~Hz}$ for LCLS and $1 \mathrm{kHz}$ for MIT XFEL) and beam energy of the machine.

Detailed design of a feedback system with $\mathrm{kHz}$ to $\mathrm{MHz}$ bandwidth for a medium energy machine is underway.

SUMMARYFor new generation of X-ray FEL the requirements on electron quality become more demanding. Besides major important parameters common for most FEL facilities including emittance, energy spread, peak current and bunch structure, some new issues in seeding type x-ray FEL facilities are taken into account from the earliest stages of machine design. In jitter studies the very precise arrival timing requirement $(\sim 20$ fs) for electron beam has become dominant factor. The optimizations have been conducted and preliminary results are very encouraging. In addition to the efforts on optimizing the linac system to minimize the sensitivity to the errors, the feedback systems will be quite helpful. We propose to use a simple system based on transverse orbit bump to quickly response the shifts of arrival timing of the beam for better synchronizations among beam, seed laser and pump lasers.

\section{ACKNOWLEDGMENTS}

We'd like to thanks R. Milner, C. Tschalar, M. Farkhondeh, W. Franklin of MIT-Bates Lab for their support to this work. We also thank O. Ilday, F. Kaertner of MIT Ultra-fast Group, J. Corlett, D. Li of LBNL for helpful discussions.

\section{REFERENCES}

[1] MIT Proposal for an x-ray FEL for NSF, 2003.

[2] LCLS CDR, SLAC-R-593, 2002, TESLA XFEL TDR, 2002.

[3] P. Emma, LCLS Lianc Design Auto-Optimization with S\&X-band RF, Arcidosso, Italy, 2000.

[4] P. Emma, Litrack code, unpublished.

[5] J. Billen, et al., Parmela, LA-UR-96-1835, 1996.

[6] M. Borland, Elegant, A Flexible SDDS-Compliant Code for Accelerator Simulation, 2003.

[7] J. Wu, et al., these proceedings.

[8] P. Emma, Talk in the FEL Beam Instrumentation Workshop, SLAC, Stanford, CA, July, 2004. 